\title{
The Effects of Early Physical Activity Compared to Early Physical Rest on Concussion Symptoms
}

\author{
Landon Lempke, Abbis Jaffri, and Nicholas Erdman
}

\begin{abstract}
Clinical Scenario: Currently, rest following concussion serves as the keystone of concussion treatment, but substantial evidence to support it is lacking. Recent literature suggests that early physical activity may be beneficial in reducing concussion symptoms which may influence clinical recovery time. Clinical Question: Does early physical activity decrease postconcussion symptoms compared to physical rest following concussion? Summary of Key Findings: A total of 5 articles were included that examined symptom duration changes at multiple time points. All 5 studies utilized follow-up time points compared to initial examination, but there was variance in the specific time points reported. Two studies employed control groups and compared strict or recommended rest to early activity or limited rest. Three studies were observational studies that directly compared baseline measurements to follow-up assessments. Clinical Bottom Line: Current evidence suggests that early physical activity in the acute phase following a concussion may decrease the time needed for symptom resolution compared to immediate rest. Strength of Recommendation: Using Centre for Evidence-Based Medicine 2011 level 3 evidence and higher, the results suggest that early physical activity during the acute phase of a concussion may decrease symptom duration; however, a lack of high-quality studies and inconsistent interventions are limitations to this recommendation.
\end{abstract}

Keywords: traumatic brain injury, rehabilitation, recovery, return to play, brain concussion

\section{Clinical Scenario}

Athletes diagnosed with sports-related concussion (SRC) have varied and complex clinical presentations that may result in symptoms that may hamper the professional, academic, or family life of athletes. An individualized approach to manage each athlete's unique symptom phenotype is necessary to prevent a protracted recovery. ${ }^{1}$ For the past several decades, physical rest has been prescribed as a mainstay for SRC management. ${ }^{1}$ More recently, rest has been divided into cognitive and physical components. Cognitive rest may include restricting daily living activities, such as school attendance, that may aggravate the symptoms. ${ }^{1}$ Physical rest recommendations consist of refraining from physical activity until the postconcussive symptoms have subsided, followed by progressive restoration of physical activity as long as the athlete is asymptomatic. ${ }^{1,2}$ Despite the widespread practice of prescribing rest following SRC, there remains a paucity of evidence supporting rest as an effective intervention. Emerging literature has indicated that prolonged periods of physical rest may be detrimental to SRC symptom recovery. ${ }^{3,4}$ Physical activity during the subacute phase ( $\geq 6 \mathrm{wk}$ following diagnosis) has been explored as a potential intervention to facilitate physical and psychological recovery from SRC as well as to improve academic outcomes in young athletes experiencing prolonged recovery. ${ }^{5}$ Although several governing bodies ${ }^{1,2}$ have recommended cognitive and physical rest following SRC, it is imperative for researchers to investigate viable postconcussion treatment strategies to appropriately manage symptoms and facilitate positive outcomes following SRC in the acute phase.

The authors conducted this work while at the University of Virginia, Charlottesville, VA. Lempke is now affiliated with the University of Georgia, Athens, GA. Lempke (lblempke@uga.edu) is corresponding author.

\section{Focused Clinical Question}

Does early physical activity decrease postconcussion symptoms compared to physical rest following concussion?

\section{Summary of Search, "Best Evidence" Appraised, and Key Findings}

- An exhaustive literature search was conducted looking for original research meeting the established inclusion criteria using the search strategy described later. The initial literature search returned 836 possible studies - 650 possible studies once duplicates were removed. All 650 studies were screened by all authors based off title for relativity, with 88 studies showing relevance. All 88 abstracts were assessed for inclusion eligibility, with 16 studies undergoing full article review.

- Of the 16 studies, only 5 studies met the inclusion criteria: 2 randomized control trials, ${ }^{6,7} 2$ prospective cohort studies, $, 8,9$ and 1 retrospective cohort study. ${ }^{10}$

- All 5 studies employed a postconcussion symptoms assessment. Four studies ${ }^{6-8,10}$ included symptom inventories with a Likert scale to indicate severity of each symptom, which included the postconcussion symptom scale, postconcussion symptom inventory, and graded symptom checklist. One study $^{6}$ used a 16-question posttraumatic complaint scale utilizing a standard visual analog scale for each question.

- Two studies utilized control groups and compared strict or recommended rest to early activity or limited rest. ${ }^{6,7}$

- Three studies were observational studies that directly compared baseline measurements to follow-up assessments. ${ }^{8-10}$

- All 5 studies used follow-up time points compared to initial examination, but there was great variation in the specific time points reported. Four studies ${ }^{7-10}$ followed up within 7 days, all 
5 studies followed up within the first month, and 1 study 6 followed out to 6 months postinjury.

\section{Clinical Bottom Line}

The current literature suggests that early physical activity in the acute phase ( $0-7 \mathrm{~d})$ following a concussion may decrease the time needed for symptom resolution compared to strict rest.

\section{Strength of Recommendation}

Using the 2011 Oxford Centre for Evidence-Based Medicine taxonomy, level 3 evidence supports the recommendation that early physical activity during the acute phase of a concussion is associated with decreased symptom severity and duration. A lack of high-quality studies and inconsistent interventions limits this recommendation.

\section{Search Strategy}

\section{Terms Used to Guide Search Strategy}

- Patient group: concussed population

- Intervention: early physical activity (acute phase of concussion)

- Comparison: physical rest

- Outcomes: symptom severity and duration, time to symptom resolution, and return to play

\section{Sources of Evidence Searched (Databases)}

- PubMed

- CINAHL

- Web of Science

- Google Scholar

\section{Search Terms}

A comprehensive search term was created to capture all articles potentially related to concussion recovery involving early physical activity and rest: (mtbi OR mild traumatic brain injury OR brain concussion OR cerebral concussion OR mild concussion OR moderate concussion OR severe concussion OR cerebral trauma OR minor head trauma OR mild head injury OR SRC OR src OR closed head injury) AND (exercise OR physical exercise OR treadmill OR bicycling OR biking OR cardio* OR aerobic activity OR walking OR buffalo concussion treadmill test OR BCTT OR controlled exercise OR graded exercise OR gradual intensity exercise OR gradual exercise OR physical exertion OR exercise therapy OR rehab* OR exertion) AND (cognitive recovery OR recovery OR rest OR mental recovery OR brain recovery OR brain rest OR physical rest) AND (symptom assessment OR symptom* OR self-reported symptom* OR head* OR dizz* OR confus* OR amnesia OR loss of consciousness OR nausea OR balance OR fatigue OR drowsiness OR sleep OR concentrat* OR fog OR slowed down OR light sensitivity OR sad* OR vomiting OR noise sensitivity OR nervous* OR memory OR numb* OR tingling OR irritable OR depress* OR vision)

\section{Limits Used}

- English language

- Original research

- Peer-reviewed academic journals

- Human population

- Published between 2000 and February 2017

\section{Inclusion and Exclusion Criteria}

\section{Inclusion Criteria}

- Comparison of physical activity to physical rest

- Comparison of strict physical rest to unrestricted physical rest

- Intervention occurred during the acute (0-7 d) phase postconcussion

\section{Exclusion Criteria}

- Failure to meet inclusion criteria and limits used

\section{Results of Search}

A total of 5 relevant studies were located and categorized as shown in Table 1 (based on Levels of Evidence, Centre for EvidenceBased Medicine, 2011). All 3 authors searched the available literature, analyzed articles for inclusion, and graded each included article using the PEDro scale. Consensus was reached upon all PEDro scoring among the 3 authors.

\section{Best Evidence}

The included studies (Table 2) were identified as the only available evidence examining early physical activity following a concussion that met our inclusion and exclusion criteria and thus serve as the best evidence.

Table 1 Summary of Study Designs of Articles Retrieved

\begin{tabular}{|c|c|c|c|}
\hline $\begin{array}{l}\text { Study design/methodology } \\
\text { of articles retrieved }\end{array}$ & $\begin{array}{l}\text { CEBM } 2011 \text { level } \\
\text { of evidence }\end{array}$ & Number located & Authors \\
\hline Randomized control trial & Level 2 & 2 & $\begin{array}{l}\text { de Kruijk et al } \\
\text { Thomas et } \mathrm{al}^{7}\end{array}$ \\
\hline Prospective cohort study & Level 3 & 2 & $\begin{array}{l}\text { Grool et } \mathrm{al}^{8} \\
\text { Buckley et } \mathrm{al}^{9}\end{array}$ \\
\hline Retrospective cohort study & Level 3 & 1 & Majerske et $\mathrm{al}^{10}$ \\
\hline
\end{tabular}




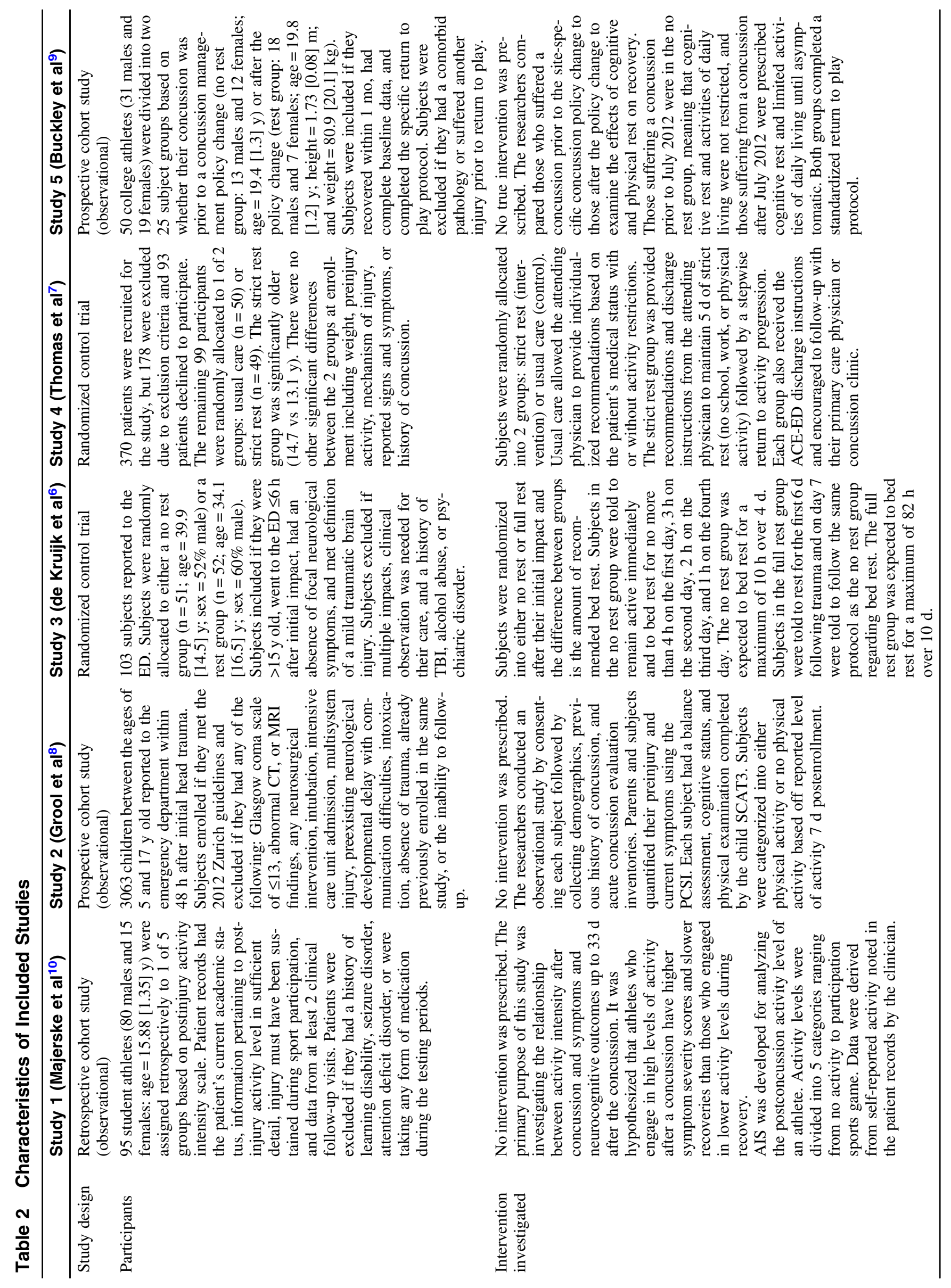




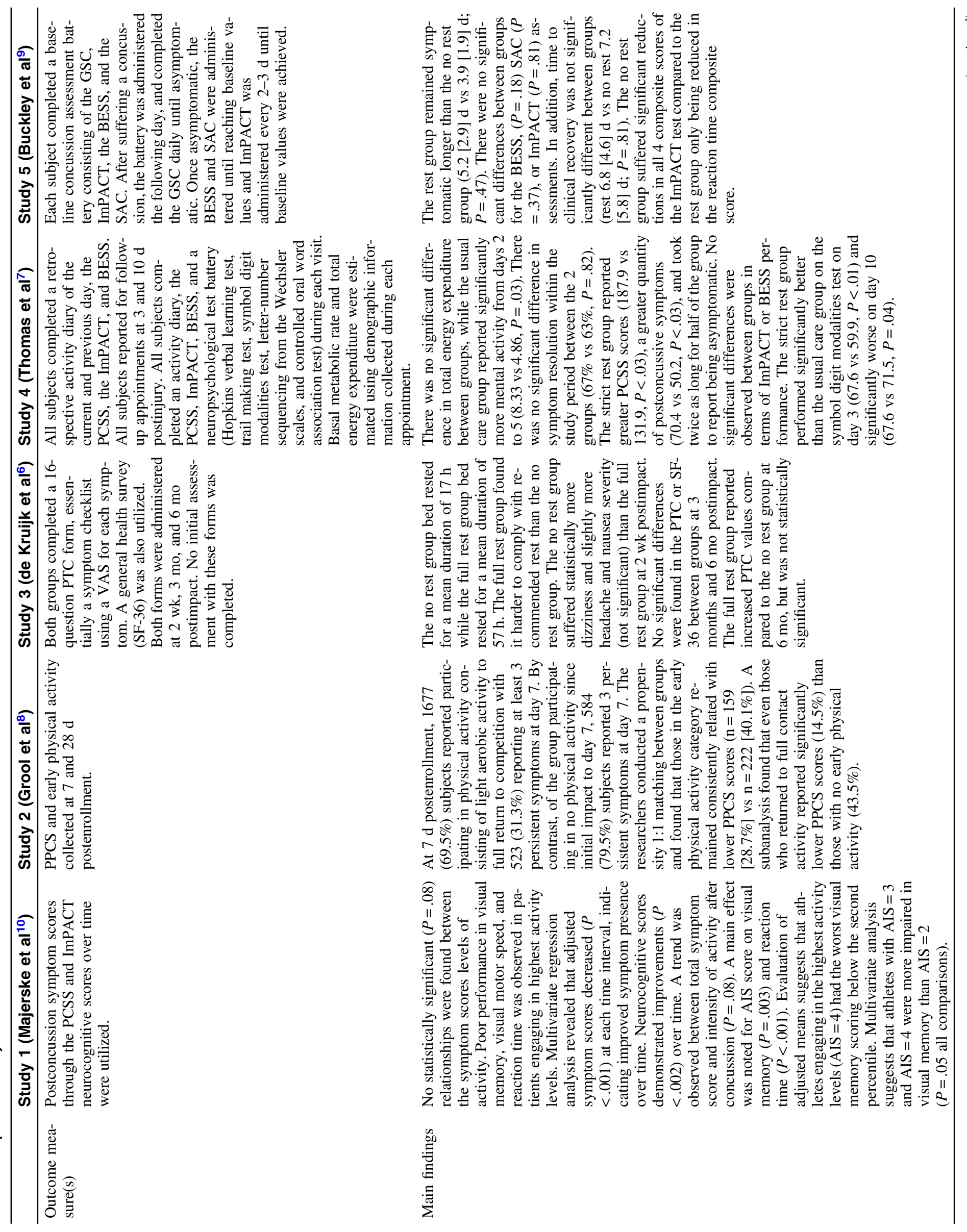




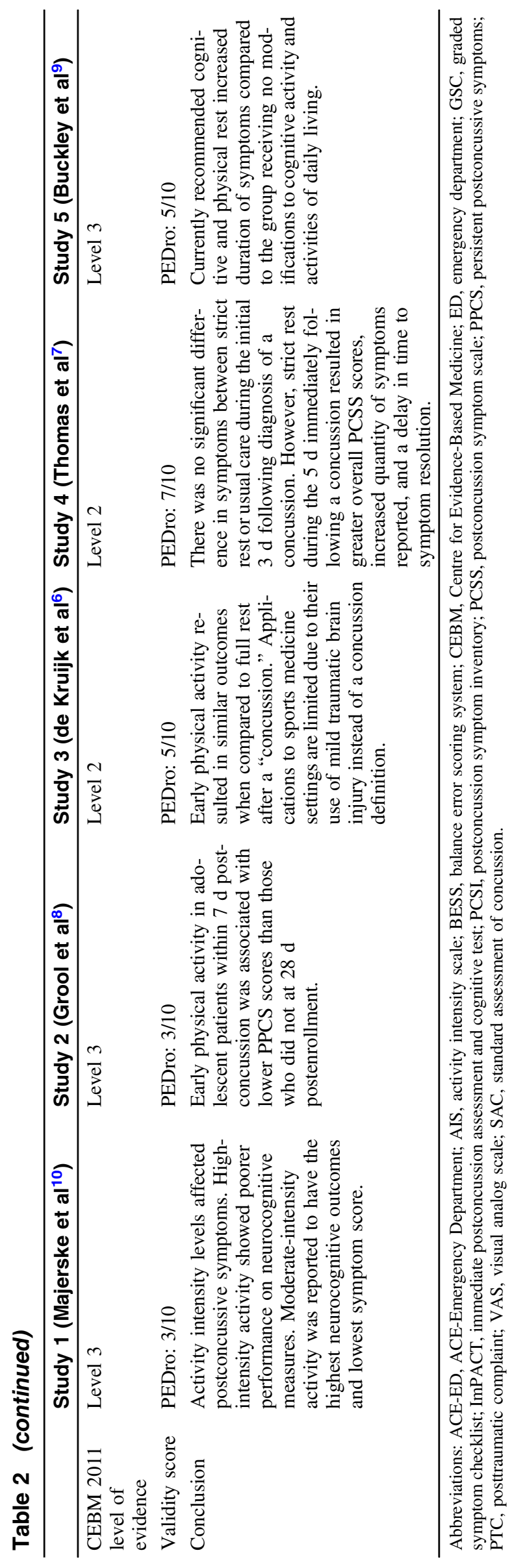




\section{Summary of Best Evidence}

\section{Implications for Practice, Education, and Future Research}

According to the 5th International Conference on Concussion in Sport, physical and cognitive rest were identified as potential avenues for improving recovery following a concussion. ${ }^{1}$ Current recommendations for the management of SRC include cognitive and physical rest for a period of 24 to 48 hours followed by assimilation back to school prior to beginning a stepwise progression of physical activity to return to sport participation. ${ }^{1}$ These conservative guidelines have been recommended to facilitate symptom resolution despite a lack of research supporting or refuting these recommendations. ${ }^{1}$ Our findings suggest that early physical activity during the acute phase of concussion (0-7 d) may decrease symptom severity and duration and ultimately influence time missed from academics or sport. Each reviewed article compared either early physical activity to strict physical rest ${ }^{7,8,10}$ or a combination of physical and cognitive rest ${ }^{6,9}$; however, interventions in the included studies were highly variable and inconsistent in the definition of concussion.

Restrictions on cognitive and physical activity were applied differently across the included studies. Two studies employed strict cognitive and physical rest for at least 5 days that included absence from school or work and bed rest following concussion. ${ }^{6,7}$ de Kruijk et $\mathrm{al}^{6}$ randomized participants to either bed rest followed by gradual activity or to stepwise activity over a 6-day period. Thomas et $\mathrm{al}^{7}$ randomized participants to either strict physical rest for 5 days or to usual care consisting of 1 to 2 days of rest followed by progressive physical activity. Thomas et $\mathrm{al}^{7}$ defined "usual care" as the clinical recommendations set by the attending physicians at the time the participant was admitted, including any cognitive or physical restrictions.

One study compared differences in concussed athletes at the college level before and after a sports medicine concussion policy change that altered their stepwise return to play guidelines. ${ }^{9}$ Participants concussed after the policy change underwent physical and cognitive rest with full academic withholding for 1 day following initial head impact, with instructions to rest in a quiet environment. Participants concussed prior to the policy change did not receive any academic accommodation and were withheld from intercollegiate athletics if symptomatic, but were not restricted on any activity outside their athletic role.

The remaining 2 studies reviewed records of patients who were diagnosed with concussions with thorough assessments and analyzed symptom and physical activity patterns based on self-reported levels of activity. ${ }^{8,10}$ Grool et $\mathrm{al}^{8}$ prospectively assessed over 2500 patients that presented to emergency departments in Canada and examined the association between their amount of physical activity and persistent symptomology 7 days postconcussion. Majerske et $\mathrm{al}^{10}$ retrospectively examined athletes who were assessed in the university's sports medicine concussion program. The researchers included only athletes who had thorough documentation of physical activity levels and academic status over at least 2 visits to determine the association of physical activity on postconcussion symptoms and neurocognitive performance.

During the first 10 days following a SRC, engaging in early physical activity was beneficial in reducing postconcussion symptoms. Participants who underwent strict physical rest resulted in a significantly longer symptom duration when compared to the physically active groups during the acute phase of concussion. ${ }^{7-10}$ Early physical activity $(0-7 \mathrm{~d})$ resulted in a $32 \%$ reduction in 3 or more symptoms' presence and significant symptom resolution at 7 days postconcussion when compared to strict physical rest. ${ }^{8}$ Strict physical rest also resulted in significantly more reported symptoms and symptom severity at 10 days postconcussion. ${ }^{7}$

Differences in symptom resolution were also observed between early physical activity and limited physical activity 1 -month postconcussion. ${ }^{6,8,10}$ Participation in early physical activity demonstrated an $11 \%$ absolute risk reduction for prolonged symptom recovery, ${ }^{8}$ although the total quantity of reported symptoms was not significantly different.6,10 Participation in high-intensity activity such as a sport game or competition was associated with increased symptom presence; however, moderateintensity activity such as school attendance and sport practice was associated with the lowest risk of symptom presence. ${ }^{10}$

Although neurocognitive and balance assessments were not the primary variables of interest in the current review, they were assessed in 3 studies. ${ }^{7,9,10}$ Limited evidence regarding neurocognitive and balance assessment was available to suggest that any treatment option was superior to another. Strict physical rest was superior within 24 hours of impact for neurocognitive and balance assessments, but was not significantly different between groups at 1 week postconcussion. ${ }^{7,9}$ Participation in school and lower intensity activity was associated with the highest neurocognitive performance, whereas sport game or competition was associated with the poorest neurocognitive performance. ${ }^{10}$ In addition, normalization of neurocognitive tests and balance assessments occurred at 1 to 2 weeks postconcussion, regardless of the subject's level of cognitive and physical rest. ${ }^{7,9}$

With the current level and quantity of research available, early physical activity appears to reduce symptom severity and duration in comparison to strict physical rest. However, this conclusion is based on limited available literature, quality of evidence, and diverse interventions studied. Our conclusions should not override current consensus guidelines ${ }^{1,2}$ but should serve as a spotlight to the best available evidence surrounding early physical activity following a concussion. Patients who are asymptomatic have not recovered physiologically, highlighting the importance of a patient-specific and evidence-based approach to achieving symptom resolution. Physical activity should be conducted in a controlled and supervised environment. The clinician should perform a daily assessment of symptom presence, and physical activity should be kept below the symptom threshold.

\section{References}

1. McCrory P, Meeuwisse W, Dvorak J, et al. Consensus statement on concussion in sport - the 5th international conference on concussion in sport held in Berlin, October 2016. Br J Sports Med. 2017;51:838847. PubMed ID: 28446457

2. Broglio SP, Cantu RC, Gioia GA, et al. National athletic trainers' association position statement: management of sport concussion. J Athl Train. 2014;49(2):245-265. PubMed ID: 24601910 doi:10. 4085/1062-6050-49.1.07

3. Silverberg ND, Iverson GL. Is rest after concussion "the best medicine?": recommendations for activity resumption following concussion in athletes, civilians, and military service members. J Head Trauma Rehabil. 2013;28(4):250-259. PubMed ID: 22688215 doi:10.1097/HTR.0b013e31825ad658 
4. McLeod TCV, Lewis JH, Whelihan K, Bacon CEW. Rest and return to activity after sport-related concussion: a systematic review of the literature. J Athl Train. 2017;52(3):262-287. PubMed ID: 28387547 doi:10.4085/1052-6050-51.6.06

5. Leddy JJ, Kozlowski K, Donnelly JP, Pendergast DR, Epstein LH, Willer B. A preliminary study of subsymptom threshold exercise training for refractory post-concussion syndrome. Clin J Sport Med. 2010;20(1):21-27. PubMed ID: 20051730 doi:10.1097/JSM. 0b013e3181c6c22c

6. de Kruijk JR, Leffers P, Meerhoff S, Rutten J, Twijnstra A. Effectiveness of bed rest after mild traumatic brain injury: a randomised trial of no versus six days of bed rest. J Neurol Neurosurg Psychiatry. 2002; 73(2):167-172. PubMed ID: 12122176 doi:10.1136/jnnp.73.2.167

7. Thomas DG, Apps JN, Hoffmann RG, McCrea M, Hammeke T. Benefits of strict rest after acute concussion: a randomized controlled trial. Pediatrics. 2015;135(2):213-223. PubMed ID: 25560444 doi:10.1542/peds.2014-0966

8. Grool AM, Aglipay M, Momoli F, et al. Association between early participation in physical activity following acute concussion and persistent postconcussive symptoms in children and adolescents. JAMA. 2016;316(23):2504-2514. PubMed ID: 27997652 doi:10. 1001/jama.2016.17396

9. Buckley TA, Munkasy BA, Clouse BP. Acute cognitive and physical rest may not improve concussion recovery time: J Head Trauma Rehabil. 2016;31(4):233-241. PubMed ID: 26394292 doi:10.1097/ HTR.0000000000000165

10. Majerske CW, Mihalik JP, Ren D, et al. Concussion in sports: postconcussive activity levels, symptoms, and neurocognitive performance. J Athl Train. 2008;43(3):265-274. PubMed ID: 18523563 doi:10.4085/1062-6050-43.3.265 